\title{
Comunicación y Transferencia en las Innovaciones Sociales. Codificación y Comunidades de Conocimiento
}

\author{
Alfonso UnCETA SATRÚstegui \\ Universidad del País Vasco-Euskal Herriko Unibertsitatea \\ alfonso.unceta@ehu.es
}

\begin{abstract}
Resumen:
Las innovaciones sociales requieren incorporar conocimiento externo a sus prácticas y reutilizar el conocimiento producido en las diferentes prácticas que la propia innovación requiere. Para que este proceso de comunicación y transferencia suceda no es suficiente con disponer de un saber hacer (dimensión tácita) sino que es preciso codificar niveles de conocimiento (dimensión explícita). Así, la articulación de conocimiento en una comunidad innovadora es un proceso de codificación de conocimientos dispersos, articulando o asimilando sistemas de codificación.
\end{abstract}

Palabras Clave: innovación social; comunicación; transferencia; conocimiento; codificación.

\section{Communication and Social Innovation Transfer. Codification and Knowledge Communities}

\begin{abstract}
Successful social innovations require the incorporation of external knowledge as well as the transformation of the knowledge produced during the development of the innovations themselves. In order for this process of communication and transference to be properly conducted it is not sufficient to have the know-how (tacit dimension) but it also necessary to encode different levels of knowledge (explicit dimension). Thus the knowledge management in an innovative community is a process of encoding scattered knowledge, either articulating or assimilating coding systems.
\end{abstract}

Key Words: social innovation; communication; transference; knowledge; coding.

\section{Referencia normalizada:}

Unceta Satrústegui, A. (2014): Comunicación y tranferencia en las innovaciones sociales. Codificación y comunicades de conocimiento. Historia y Comunicación Social. Vol. 19. Núm. Especial Marzo. Págs. 763-776.

Sumario: 1. Introducción. 2. Modelos de conocimiento. 3. Tipos de Conocimiento. 4. La Innovación Social: Codificación de Conocimiento en entornos comunitarios. 5. Conclusiones. 6. Referencias Bibliográficas. 


\section{Introducción}

La capacidad de crear, comunicar y transferir conocimiento en una comunidad es dependiente de la combinación e integración de conocimientos heterogéneos y, en definitiva de la acción comunicativa que expresa y explica dicha combinatoria. Estas dinámicas remiten a la codificación de conocimiento. La codificación sugiere la creación de códigos, es decir, un conjunto de signos y reglas de combinación que estructura la producción de mensajes y su interpretación con sentido en una comunidad dada.

La codificación de conocimiento constituye un elemento clave para la comunicación de las innovaciones sociales (WESTLEY y ANTANDZE, 2010) y su transferencia hacia otros contextos sociales (KIRAT y LUNG, 1999). En gran medida el discurso sobre la innovación ha enfatizado el conocimiento y el aprendizaje colectivo (collective learning) (LAWSON y LORENZ, 1999) y por tanto las comunidades de conocimiento como contextos privilegiados no solo para el aprendizaje en sí mismo sino para crear, transformar, comunicar y transferir conocimiento. (WENGER, 1998; COAKES y CLARKE, 2006; ECHEVERRÍA, ALONSO, y OIARZABAL, 2011).

Ahora bien, una parte del conocimiento (codificado) se encuentra almacenada en un libro de códigos validados (o considerados válidos) por una comunidad. Este proceso de validación es una construcción social, se trata de un conjunto de mensajes e informaciones "consensuadas" por la comunidad, no reflejan un conocimiento transparente y verdadero de lo real sino un proceso de acuerdo inestable (GRIMM, 2006). Complementariamente, otra parte de conocimiento (tácita) no es acumulable en el libro de códigos pero es una dimensión clave y dinámica para producir nuevos mensajes y para interpretar los existentes. Lo relevante desde la perspectiva de la comunicación y la transferencia de conocimiento es que el libro de códigos es el proceso interactivo mediante el cual una comunidad crea y define conocimiento (elabora su terminología, modelos, conceptos, etc.), algo que deviene fundamental tanto para interpretar como para escribir mensajes e inscribir conocimiento. (COWAN, DAVID, y FORAY, 2000) En un sentido radical, el libro de códigos "es" la propia comunidad que condensa y amalgama sus acuerdos (consensos) explicitándolos en dicho libro.

Supuesto lo anterior, conviene recordar que la naturaleza del conocimiento ha sugerido debates entre distintas perspectivas y ha generado una extensa literatura filosófica. La revisión de estas posiciones epistemológicas no constituye el objetivo de este artículo. Doy por supuesto que el conocimiento es de naturaleza compleja y no existen consensos claros en torno a muchas de sus dimensiones constitutivas. Sin embargo, mi interés se concentra en la forma en que las comunidades de conocimiento vinculadas a los procesos de innovación social codifican conocimiento y construyen un conjunto de signos y reglas que, combinados, organizan mensajes, y formalizan códigos que pueden ser elucidados por aquellos que aun no habiendo elaborado el código pueden aprenderlo, interpretarlo, modificarlo y desarrollarlo en diferentes contextos (temporales y espaciales). Esta circunstancia resulta crucial para 
comprender los procesos de comunicación y transferencia en las innovaciones sociales.

\section{Modelos de conocimiento}

En líneas generales, desde la perspectiva de los procesos de creación, adecuación, comunicación, y transferencia de conocimiento, podemos identificar dos cuerpos conceptuales que exploran estas relaciones: el modelo cognitivo y el modelo comunitario (BRESNEN, EDELMAN, NEWELL, SCARBROUGH, y SWAN, 2003; HANSEN, NOHRIA, y TIERNEY, 1999; RUUSKA y VARTIAINEN, 2005).

El modelo cognitivo asume una perspectiva racionalista del conocimiento según la cual la naturaleza es externa (separación sujeto - objeto) y el conocimiento está aislado de la experiencia. Conocer es un acto intelectual de interpretación de lo real, y como sugiere Latour (1993) la verdad se explica por la naturaleza y lo falso por lo social (las intuiciones, las experiencias, etc.). De esta manera la verdad se deduce a partir de un pensamiento racional, mediante axiomas. Así, el conocimiento es un proceso intelectual que emplea conceptos, leyes y teorías para identificar la verdad.

Esta perspectiva racionalista puede definirse como la reducción del conocimiento a datos e información (datos organizados en mensajes) según un modelo lineal de conocimiento: los datos se transforman en información, la información en conocimiento que se confronta con la visión del mundo (creencias, juicios y valores) (ANCORI, BURETH, y COHENDET, 2000; COHENDET, CREPLET, y DUPOÜET, 2000; DUGUID, 2005). Lo que aquí se enfatiza es el proceso de codificación de la información incrementando el conocimiento a partir de acumular datos que se transforman en información: mientras mayor información mayor desarrollo del conocimiento a partir de mayor capacidad de integración de información heterogénea y capacidad combinatoria (ALAVI y LEIDNER, 2001; BARREAU, 1995; FRED, JAMES, y JAMES, 1991).

Para esta corriente de pensamiento el conocimiento constituye esencialmente acumulación de información en el marco de un flujo de mensajes (ANCORI, BURETH, y COHENDET, 2000) donde lo relevante es el desarrollo de un eficaz sistema de gestión de la información (capacidad creciente de integración y complejidad) basada en un eficiente mecanismo de tratamiento de datos como proceso de formación de mensajes y conocimiento (ALAVI y LEIDNER, 2001). Consecuentemente, se entiende que el proceso de codificación de conocimiento está basado en complejos modos de procesar, integrar y combinar información.

El modelo comunitario, por su parte, acentúa el carácter social e interactivo de la creación, codificación, comunicación y transferencia del conocimiento, destacando la importancia de la dimensión tácita del conocimiento y la naturaleza colectiva de su codificación ( BRESNEN, EDELMAN, NEWELL, SCARBROUGH, y SWAN, 
2003; HANSEN, NOHRIA, y TIERNEY, 1999; NEWELL, BRESNEN, EDELMAN, SCARBROUGH, y SWAN, 2006; NONAKA, 1994). Esta perspectiva asume una posición constructivista del conocimiento según la cual el conocimiento es el resultado de una "interpretación" y "consenso" que se produce como fruto de un proceso social, reflexivo e interactivo de aprendizaje, difícil de comunicar y transferir y altamente complejo de codificar (ANCORI, BURETH, y COHENDET, 2000; COOK y BROWN, 1999).

En este modelo, el proceso de creación de conocimiento supone el desarrollo de comunidades que comparten ideas, información y conocimiento a partir de mecanismos colaborativos de trabajo (WENGER, 1997; WENGER, MC DERMOTT, y SNYDER, 2007), en las que los procesos comunicativos que facilitan la asociación de las personas con las personas (HANSEN, NOHRIA, y TIERNEY, 1999) devienen vitales. En este caso, compartir significa que los agentes desarrollan una práctica (una actividad) en común y crean conjuntamente un sendero particular de aprendizaje razón por la cual la producción, comunicación y transferencia del conocimiento se encuentra siempre "situada", es decir, que está fuertemente influenciada por el contexto social en el que se produce la práctica (BRESNEN, EDELMAN, NEWELL, SCARBROUGH, y SWAN, 2003; WENGER, 1998).

En la figura $\mathrm{N}^{\mathrm{o}} 1$ se ofrece un modelo interactivo de conocimiento basado en Ancori y colaboradores ( ANCORI, BURETH, y COHENDET, 2000). En el modelo se observa, en primer lugar, que los "datos" son reemplazados por estímulos ("la naturaleza no habla a través de datos"). Los estímulos no están organizados (como los datos) pero pueden ser interpretados gracias a la existencia de marcos cognitivos, que facilitan el proceso de estructuración (clasificación e interpretación de los estímulos) y pueden ser percibidos gracias a una visión del mundo (que asigna valores y juicios) (AKGÜN, LYNN, y BYRNE, 2003).

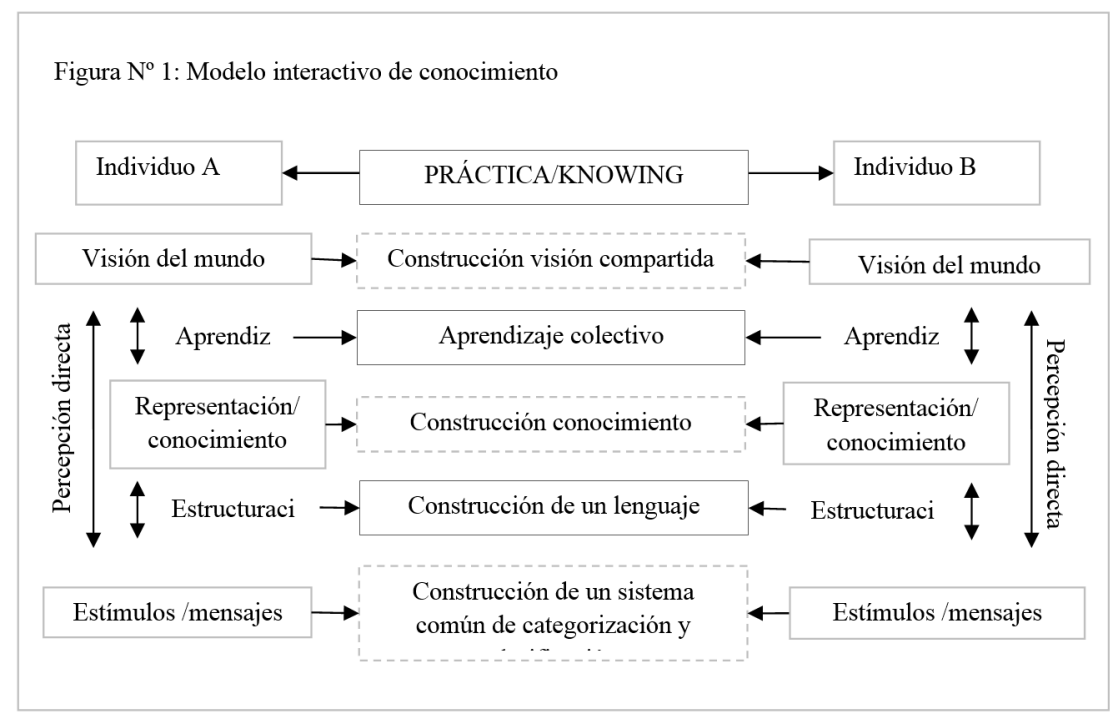


En segundo lugar, el conocimiento y la representación sugieren que la estructuración de estímulos se produce en un primer momento como una representación y en un segundo momento como conocimiento (sedimentación de representaciones). En tercer lugar, la visión del mundo interactúa con el conocimiento creando espacios de aprendizaje. En cuarto lugar, este proceso en los individuos no se produce de manera aislada sino en procesos nuevos y colectivos (interactivos) de comunicación y transferencia (GARAVAN y MCCARTHY, 2008; KNAPP, 2010).

En consecuencia con lo anterior, la interpretación de los estímulos queda estructurada en sistemas categoriales compartidos (lenguaje común) (BARR, 2004; BICCHIERI, 1988). Se pueden interpretar los estímulos de manera compartida porque las personas interactúan construyendo sentido, una visión compartida del mundo, aprendiendo y comunicándose colectivamente (interactivamente) (AKGÜN, LYNN y BYRNE, 2003; AYAS y ZENIUK, 2001). Pero esos procesos interactúan en la práctica. La práctica implica hacer cosas informadas por el conocimiento y la visión del mundo (COOK y BROWN, 1999) en un contexto particular, específico. Razón por la que las prácticas sugieren la configuración de aprendizajes situados (BROWN, COLLINS, y DUGUID, 1989; DUGUID, 2005) y la visión del mundo en contexto (enculturación) (BLACKLER, 1995).

Así, la noción de knowing (para que la que no existe una traducción fiel al castellano) significa que el conocimiento y su interacción con la visión del mundo (aprendizaje, que es la manera de acumular conocimiento) no es algo que "se use" para la práctica o algo "necesario" para la práctica sino que es "parte" de la práctica misma (acción) (COOK y BROWN, 1999: 386). Una dimensión tácita del conocimiento, basada en procesos comunicativos específicos, que solamente se activa en la práctica (POLANYI, 1967) y sólo es en ésta donde se ejercen (no se posee, sino que se ejerce).

Los dos enfoques reseñados (cognitivo y comunitario) llevan implícitos dos grandes visiones sobre el aprendizaje, la comunicación y la transferencia: en el primer caso estamos ante procedimientos de adquisición, comunicación y transferencia de conocimiento que resultan de un proceso individual; en el segundo, adquisición, comunicación y transferencia de conocimiento se entienden como procesos participativos que descansan en su carácter social, interactivo, a través de una práctica.

En el primer caso, el conocimiento se adquiere, se posee, se comunica y se transfiere a través de procesos en los que predomina el conocimiento explícito (codificado) que está basado en información. En el segundo caso, el conocimiento se construye en el proceso de interacción mismo y es contextual a ese proceso comunicativo y no existe antes de él, aspecto que destaca el carácter co-construido, tácito y situacional del conocimiento. Estas dos corrientes difieren en los mecanismos de aprendizaje, comunicación y transferencia, de acuerdo a un patrón de pasividad o actividad. Así en la primera perspectiva los individuos se enriquecen con conocimientos, es decir, son propietarios o poseen "algo" nuevo mientras que en la segunda perspectiva los individuos construyen comunidades, elaboran entornos de aprendizaje, comunicación 
y transferencia. (COOK y BROWN, 1999; LAVE y WENGER, 1991; SFARD, 1998).

Aún cuando el análisis anterior puede conducir a una visión dicotómica de los modelos cognitivo y comunitario, es necesario advertir que, en general, el aprendizaje, la comunicación y la transferencia basados en la adquisición de conocimiento desde la perspectiva individual no desconoce la dimensión social del conocimiento. Asimismo, el aprendizaje, la comunicación y la transferencia basados en la práctica participativa tampoco desconoce el efecto individual en tanto adquisición y almacenamiento de conocimiento en los individuos (SFARD, 1998). Sin embargo y a nuestros efectos analíticos, la contraposición de ambas perspectivas permite poner el énfasis en la dimensión individual o colectiva de los procesos de aprendizaje, comunicación y transferencia de conocimiento.

\section{Tipos de Conocimiento}

Si bien es cierto que existen distintas formas de clasificar e identificar los tipos de conocimiento ${ }^{1}$, no lo es menos que la distinción entre la dimensión tácita y la dimensión explicita del conocimiento realizada por Polanyi (1967) ha sido pionera para repensar las maneras en las que se crea, comunica y transfiere conocimiento. Como ha sugerido Polanyi "sabemos más de lo que podemos decir" (Polanyi, 1967:4). De esta manera Polanyi busca poner de relieve lo que él denomina una dimensión tácita del conocimiento, al sugerir que el conocimiento tácito es intuitivo, no verbalizable y desarticulado. Se trata de un saber-hacer (know how) y es resultado de un proceso de acumulación basado en la experiencia y en la práctica. No es un conocimiento orientado hacia la práctica sino es la práctica misma (tacit knowing).

De acuerdo a lo anterior, en la medida en que el conocimiento tácito es intuitivo, desarticulado y situacional (se expresa en un acto), los factores contextuales facilitan u obstaculizan su desarrollo y expresión. El conocimiento tácito es muy complejo de codificar, comunicar y transferir. Dado que el conocimiento tácito está situado y circunscripto a campos de experiencias determinados puede ser aprendido, co-creado, comunicado y transmitido gracias a una interacción sistemática y directa en torno a una práctica específica y regular (COHENDET y MEYER-KRAHMER, 2001; COWAN, DAVID, y FORAY, 2000; D’EREDITA y BARRETO, 2006;

1 Por ejemplo, Winter (1987: 163) distingue diferentes tipos de conocimiento: (a) Complejo vs. simple, (b) enseñable vs. no enseñable, (c) observable vs. no observable. Por su parte, Lundvall y Johnson (1994) diferencian cuatro tipos de conocimiento: saber qué, saber por qué, saber-hacer y saber quién. Define así estos tipos de conocimiento: (a) Know-what: refers to knowledge about "facts"; (b) Knowwhy: refers to scientific knowledge of the principles and laws of nature; (c) Know-how: refers to skills or the capability to do something; (d) know-who: involves information about who knows what and who knows how to do what (LUNDVALL y JOHNSON, 1994: 27). 
JOHNSON, LORENZ, y LUNDVALL, 2002), es decir, en el marco de una comunidad (BRESNEN, EDELMAN, NEWELL, SCARBROUGH, y SWAN, 2003; COHENDET, CREPLET, y DUPOÜET, 2000; DUGUID, 2005; PINCH, ROB, y NIGEL, 2009).

Por otro lado, el conocimiento explícito se expresa en modelos y procesos formalizados que se estructuran en diferentes soportes (libros, manuales, documentos, patentes, diseños, software, etc.), su comunicación y transferencia es menos dependiente del contexto en el que ha sido producido ${ }^{2}$ y puede ser intercambiado en diferentes momentos y entre diferentes espacios (y soportes). Su mecanismo de adquisición y transferencia está asociado a procesos lógicos y adquiridos mediante la educación formal y estudios (ANCORI, BURETH, y COHENDET, 2000; COHENDET y MEYER-KRAHMER, 2001; JOHNSON, LORENZ, y LUNDVALL, 2002; LAM, 1997).

Uno de los elementos centrales sugeridos por Polanyi (1967) es que el conocimiento explícito precisa del conocimiento tácito para ser utilizado. En rigor, ambas son "dimensiones" del mismo conocimiento y no se encuentran contrapuestas ni separadas una de la otra para poder operar en la práctica. (Polanyi, 1967: 17). Que el conocimiento tácito y el explicito se impliquen recursivamente es fuente de debate y controversia (COWAN, DAVID, y FORAY, 2000; DUGUID, 2005; FERNIE, GREEN, WELLER, y NEWCOMBE, 2003; GOURLAY, 2006; TSOUKAS, 2003). Así, una posición sugiere que el conocimiento tácito puede codificarse (aún cuando es una tarea compleja y difícil). (COWAN, DAVID, y FORAY, 2000; NONAKA, 1994; SAVIOTTI, 1998), mientras que la otra mantiene que el conocimiento tácito interactúa con el conocimiento explícito pero que este proceso de interacción no es de codificación ( JOHNSON, LORENZ y LUNDVALL, 2002; TSOUKAS, 2003).

Ancori, Bureth, y Cohendet (2000), sugieren una relación una relación dinámica entre el conocimiento explícito y el conocimiento tácito y sus expresiones individuales y colectivas. Tal relación puede sintetizarse como sigue:

- El conocimiento explícito de carácter individual es objetivable, adquirido y codificado, y permite utilizar métodos racionales para generar la comunicación y transferencia de dicho conocimiento.

2 En rigor, hay autores que sostienen que esta afirmación es relativa, puesto que para incorporar conocimiento codificado es preciso disponer de un contexto de integración de conocimientos que implica compartir y haber asimilado parte del paradigma en el marco del cual "ese" conocimiento ha sido codificado. Este paradigma es difícil de comprender, reproducir e interpretar cuando la unidad receptora se encuentra alejada de la fuente original de codificación (SORENSON, RIVKIN, y FLEMING, 2006). En esta línea Ancori y coautores sugieren: "Particular pieces of information can be understood only in the context of a given type of knowledge. New knowledge, for example relative to radical innovation, creates new information. However, this information can only be understood and used by those who possess the new knowledge (...) In summary, knowledge is a correlational and a retrieval/ interpretative structure, and it has a local character"(ANCORI, BURETH, y COHENDET, 2000: 265) 
- El conocimiento explícito de carácter colectivo guía las prácticas sociales y organizacionales (leyes, normas etc.). Tales prácticas están codificadas y su comunicación y transferencia es accesible a todas las personas de una comunidad: código explícito.

- El conocimiento tácito de carácter individual puede denominarse "conocimiento automático" y se estructura en lo que cabe denominar competencias. Puede decirse que funcionan solamente cuando son requeridas en un proceso de producción, comunicación o transferencia de conocimiento.

- El conocimiento tácito de carácter colectivo, no se encuentra objetivado y se expresa en reglas tácitas de funcionamiento (por ejemplo la confianza), reglas de conducta etc. Se trata, por decirlo así, de prácticas silenciosas que generar procesos singulares de comunicación y transferencia.

\section{La Innovación Social: Codificación de Conocimiento en entornos comunita- rios}

La innovación social es una actividad estratégica para el desarrollo de comunidades de conocimiento. Como bien ha señalado Hochgerner (2009) la generación, adaptación, comunicación y difusión de las innovaciones sociales no ocurren, por definición, en entornos individuales sino en entornos sociales configurados. ${ }^{3}$ De la misma manera, la generación, comunicación y transferencia de innovaciones sociales no puede lograrse a través de individuos actuando en solitario. Además, como voy a explicar, las comunidades de conocimiento facilitan la codificación de conocimiento y su almacenamiento en un libro de códigos puesto que, el libro de códigos "es" la propia comunidad, algo que ya he señalado.

Así, la creación de conocimiento en una comunidad configura un proceso de formación de mensajes, información y conocimiento en un formato de "código abierto" (para la comunidad) en la que se estructura un sistema social de interacciones. En este sentido, una comunidad de conocimiento es una red epistémica, es decir, es comunidad y red a la vez. La comunidad estructura el libro de códigos (sistema de reglas compartidas y estructurantes de nuevos mensajes) mientras que la red (que pone en interacción a los individuos) desarrolla los mensajes dentro del libro.

En la medida que la comunidad codifica conocimiento, organiza un libro de códigos que en el origen de la comunidad no está escrito. De esta manera, el libro de códigos se va escribiendo en tanto el conocimiento se va codificando dado que el libro de códigos es un mecanismo de acumulación y almacenamiento de documentos

3 Esta es una cuestión compartida por la gran mayoría de autores que se han interesados por la innovación social. Ver a modo de ejemplo ECHEVERRÍA, (2008); GURRUTXAGA, y ECHEVERRIA, (2012); CASTRO SPILA, y UNCETA, (2013.; CASTRO SPILA, ROCCA, e IBARRA, (2008); MULGAN, (2006); MURRAY, R., CAULIER-GRICE, y MULGAN, (2010). 
que facilita el proceso recursivo de la codificación (codificación-decodificación) por cuanto es fuente de consulta (al modo de un diccionario), interpretación, comunicación y transferencia. Este proceso de ir desarrollando el libro de códigos implica crear un vocabulario y modelos operativos para producir y "escribir" conocimiento. Y también para comunicarlo y transferirlo. El libro de códigos además permite estabilizar el conocimiento (con sus conceptos, jerga, lenguajes, etc.). Desde este punto de vista los procesos de creación, comunicación y transferencia de conocimiento son una relación dinámica entre estabilización y desestabilización del conocimiento, lo que desarrolla progresivamente el libro de códigos.

Ahora bien, la codificación del conocimiento en una comunidad es un proceso de articulación de conocimientos dispersos en orden a crear el libro de códigos: modelos, lenguaje (conceptos) y escritura de mensajes (documentos) y relaciones. De esta manera, la codificación es un proceso recursivo y dinámico del conocimiento sobre el conocimiento mismo que tiene más probabilidades de suceder cuando se dispone de conocimiento acumulado (libro de códigos) y experiencia en aprender del conocimiento nuevo (COHEN y LEVINTHAL, 1990). En tanto proceso recursivo y acumulativo la codificación del conocimiento no está separada de sus condiciones de codificación, es decir, que es un proceso situado social, temporal y espacialmente (COHENDET y MEYER-KRAHMER, 2001; LAM, 2000), razón por la cual, expresa un sendero de aprendizaje único y difícilmente imitable. Una comunidad de conocimiento desarrolla sus capacidades de codificación en la medida en que desarrolla sus capacidades de aprendizaje colectivo.

De ahí que las comunidades de conocimiento sean caracterizadas como un espacio social capaz de movilizar conocimientos individuales que se articulan en un proceso colectivo e integran en el libro de códigos una heterogeneidad de información y conocimiento que se refiere a las causas o leyes (de la naturaleza o de lo social), implicaciones empíricas o ilustraciones sobre hechos derivados de las causas, así como estructuras procedimentales y relacionales para la resolución de problemas en que son los que organizan una comunidad de conocimiento específica (JOHNSON, LORENZ, y LUNDVALL, 2002; LUNDVALL y JOHNSON, 1994).

Finalmente, existe comunidad en la medida en que otros integrantes (diferentes de aquellos que han escrito mensajes en el libro de códigos) puedan acceder al conocimiento integrado en el libro de códigos y pueden interpretarlo y decodificarlo con sentido, lo que implica no sólo conocimiento explicito sino tácito, y procesos específicos de comunicación y transferencia.

\section{Conclusiones}

He tratado de explicar que las innovaciones sociales se producen y reproducen entornos localizados de conocimiento y tienen vocación de generar espacios que promuevan el aprendizaje, intercambio, comunicación y transferencia de cono- 
cimiento. Asimismo, los procesos de innovación social se estructuran en torno al conocimiento ya adquirido en combinación con nuevo conocimiento. Dadas las características anteriores, las innovaciones sociales tienden a promover comunidades de conocimiento que, a su vez, como ya he reiterado, facilitan la codificación de conocimiento (libro de códigos).

La secuencia que acabo de describir resulta crucial para comprender la manera en que, en el caso de las innovaciones sociales, la comunicación y transferencia de conocimiento está directamente vinculada a las dinámicas de las comunidades de conocimiento y, en particular, al conocimiento integrado en el libro de códigos. En síntesis, el carácter crucial de estas comunidades y los procesos de codificación que generan, para la comunicación y transferencia de conocimiento en el caso de las innovaciones sociales puede identificarse en los siguientes rasgos:

1. El efecto incremental del conocimiento que tiende a producirse es debido, en gran medida, a la codificación de conocimiento que facilita su circulación y transferencia. Induce la posibilidad de de adquirir, interpretar, procesar y transformar el conocimiento existente en el libro de códigos

2. La codificación del conocimiento favorece el aprendizaje y la transferencia a otros integrantes comunitarios (diferentes de aquellos que han escrito mensajes en el libro de códigos) en tanto puedan acceder al conocimiento integrado en dicho libro.

3. La codificación de conocimiento desarrolla competencias (generativas) de codificación en los individuos que participan de la comunidad y que facilita la creación de nuevas comunidades (efecto expansivo de la formación para la crear nuevas comunidades).

Por último, conviene recordar que, en el análisis de los entornos comunitarios, lo que ha sido conceptualizado como "capacidad de absorción de conocimiento" es un concepto relacional que define la habilidad de las organizaciones para identificar, asimilar, transformar, comunicar y transferir conocimiento. (COHEN and LEVINTHAL, 1990). Sin duda, la codificación-descodificación de conocimiento en el libro de códigos que produce una comunidad, y los consiguientes procesos de comunicación y transferencia, dependen en gran medida de su capacidad de absorción de conocimiento.

\section{Bibliografía}

\subsection{Libros}

COAKES, E. Y CLARKE, S. (2006): Encyclopedia of Communities of Practice in Information and Knowledge Management. Hershey, PA, IGI Publishing. 
ECHEVERRÍA, J., ALONSO, A. Y OIARZABAL, P. J. (2011): Knowledge Communities. Centre for Basque Studies. Reno. University of Nevada.

GURRUTXAGA, A. Y ECHEVERRIA, J. (2012): La luz de la luciérnaga. Diálogos de innovación social. Madrid. Plaza y Valdés Editores

LATOUR, B. (1993). Nunca hemos sido modernos. Ensayo de Antropología Simétrica. Madrid: Debate.

LAVE, J., Y WENGER, E. (1991). Situated learning: Legitimate peripheral participation. Cambridge, England: Cambridge University Press.

MURRAY, R., CAULIER-GRICE, J., Y MULGAN, G. (2010). The Open Book of Social Innovation. London: NESTA.

POLANYI, M. (1967). The Tacit Dimension. London: Routledge and Kegan Paul.

WENGER, E. (1997). Communities of practice: Learning, meaning, and identity. Cambridge.: Cambridge University Press.

WENGER, E., MC DERMOTT, R., Y SNYDER, W. (2007). Cultivating communities of practice: A guide to managing knowledge. Harvard Business School Press.

6.2 Capítulos de Libro

CASTRO SPILA, J. Y UNCETA, A. (2013). "Sistemas localizados de conocimiento: lecciones para la innovación social". En Villafañe, J. (Dtor) (2013): La comunicación empresarial y la gestión de los intangibles en España y Latinoamérica. Madrid. Pearson. P. 245-266.

ECHEVERRÍA, J. (2008). "Aportaciones preliminares a los estudios de innovación social”. En Mendiola I. (edit.) (2008): Textos y pretextos para repensar lo social. Bilbao. UPV-EHU. p. 81-96.

PINCH, S., ROB, K., Y NIGEL, T. (2009). "Knowledge Communities". En International Encyclopedia of Human Geography Oxford: Elsevier. P. 25-30.

TSOUKAS, H. (2003). “Do we really understand tacit knowledge?” En M. a. L. Easterby-Smith, M.A. (Eds) (Ed.), The Blackwell handbook of organizational learning and knowledge management. Oxford: Blackwell Publishing Ltd. P. 410-427.

WINTER, S. G. (1987). "Knowledge and competence as strategic assets". En D. Teece (Ed.), The Competitive Challenge. Cambridge, MA: Ballinger Publishing. p.36-42

\subsection{Revistas Científico-Profesionales}

AKGÜN, A. E., LYNN, G. S., y BYRNE, J. C. (2003). “Organizational Learning: A Socio-Cognitive Framework”. Human Relations, 56(7), p. 839-868.

ALAVI, M., y LEIDNER, D. E. (2001). "Review: Knowledge Management and Knowledge Management Systems: Conceptual Foundations and Research Issues." En MIS Quarterly, 25(1), p. 107-136.

ANCORI, B., BURETH, A., y COHENDET, P. (2000). "The economics of knowledge: the debate about codification and tacit knowledge." En Industrial and Corporate Change, 9(2), p. 255-287.

AYAS, K., y ZENIUK, N. (2001). "Project-Based Learning: Building Communities of Reflective Practitioners" En. Management Learning, 32(1), p. 61-76. 
BARR, D. J. (2004). “Establishing conventional communication systems: Is common knowledge necessary?" En Cognitive Science, 28(6), p. 937-962.

BARREAU, D. K. (1995). "Context as a factor in personal information management systems." En Journal of the American Society for Information Science, 46(5), p. 327-339.

BICCHIERI, C. (1988). "Backward Induction without Common Knowledge". En PSA: Proceedings of the Biennial Meeting of the Philosophy of Science Association, 1988, p. 329-343.

BLACKLER, F. (1995). "Knowledge, Knowledge Work and Organizations: An Overview and Interpretation”. En Organization Studies, 16(6), p. 1021-1046.

BRESNEN, M., EDELMAN, L., NEWELL, S., SCARBROUGH, H., y SWAN, J. (2003)." Social practices and the management of knowledge in project environments". En International Journal of Project Management, 21(3), p. 157-166.

BROWN, J. S., COLLINS, A., y DUGUID, P. (1989). "Situated Cognition and the Culture of Learning”. En Educational Researcher, 18(1), p. 32-42.

CASTRO SPILA, J. ROCCA, L. e IBARRA, A. (2008). "Transferencia de conocimiento en las empresas de la Comunidad Autónoma del País Vasco: capacidad de absorción y espacios de interacción de conocimiento". En Arbor, Vol. CLXXXIV (732), p.653-675

COHEN, W. M., y LEVINTHAL, D. (1990). “Absorptive capacity: A new perspective on learning and innovation". En Administrative Science Quarterly, 35(1), p. 128-152.

COHENDET, P., y MEYER-KRAHMER, F. (2001). "The theoretical and policy implications of knowledge codification". En Research Policy, 30(9), p. 15631591.

COOK, S. D. N., y BROWN, J. S. (1999). "Bridging Epistemologies: The Generative Dance Between Organizational Knowledge and Organizational Knowing". En Organization science 10(4), p. 381-400.

COWAN, R., DAVID, P., y FORAY, D. (2000). “The explicit economics of knowledge codification and tacitness". En Industrial and Corporate Change, 9, p. 211-253.

D’EREDITA, M. A., y BARRETO, C. (2006). "How Does Tacit Knowledge Proliferate? An Episode-Based Perspective". En Organization Studies, 27(12), p. 18211841.

DUGUID, P. (2005). “The art of knowing": social and tacit dimensions of knowledge and the limits of the community of practice. En The Information Society, 21(2), p. 109-118.

FERNIE, S., GREEN, S. D., WELLER, S. J., y NEWCOMBE, R. (2003). “Knowledge sharing: context, confusion and controversy". En International Journal of Project Management, 21(3), p. 177-187.

FRED, N., JAMES, C. B., y JAMES, C. W. (1991).” Information systems management issues for the 1990s". En MIS Q., 15(4), p. 475-500.

GARAVAN, T. N., y MCCARTHY, A. (2008). "Collective Learning Processes and Human Resource Development". En Advances in Developing Human Resources, 10(4), p. 451-471. 
GOURLAY, S. (2006). “Conceptualizing Knowledge Creation: A Critique of Nonaka’s Theory”. En Journal of Management Studies, 43, p. 1415-1436.

GRIMM, S. R. (2006). "Is Understanding a Species of Knowledge?" En The British Journal for the Philosophy of Science, 57(3), p. 515-535.

HANSEN, M., NOHRIA, N., y TIERNEY, T. (1999). "What's your strategy for managing knowledge?" En Harvard Business Review, p. 106-116.

JOHNSON, B., LORENZ, E., y LUNDVALL, B. A. (2002).'Why all this fuss about codified and tacit knowledge?" En Industrial and Corporate Change, 11(2), p. 245-262.

KIRAT, T., y LUNG, Y. (1999). "Innovation and Proximity: Territories as Loci of Collective Learning Processes". En: European Urban and Regional Studies, 6, (1) p. 27-38.

KNAPP, R. (2010). “Collective (Team) Learning Process Models: A Conceptual Review". En Human Resource Development Review, 9(3), p. 285-299.

LAM, A. (1997). "Embedded Firms, Embedded Knowledge: Problems of Collaboration and Knowledge Transfer in Global Cooperative Ventures". En Organization Studies, 18(6), p. 973-996.

LAWSON, C. y LORENZ, E. (1999). “Collective Learning, Tacit Knowledge and Regional Innovative Capacity". En Regional Studies 33, p. 305-317.

LUNDVALL, B. A., y JOHNSON, B. (1994). “The Learning Economy”. En Journal of Industry Studies, 1(2), p. 23-42.

MULGAN, G. (2006). "The process of social innovation. Innovations: Technology, Governance, Globalization”, En 1(2), p. 145-162.

NEWELL, S., BRESNEN, M., EDELMAN, L., SCARBROUGH, H., y Swan, J. (2006). "Sharing Knowledge Across Projects: Limits to ICT-led Project Review Practices". En Management Learning, 37(2), p. 167-185.

NONAKA, I. (1994). "A Dynamic Theory of Organizational Knowledge Creation". En Organization Science, 5(1), p. 14-37.

RUUSKA, I., y VARTIAINEN, M. (2005). "Characteristics of knowledge sharing communities in project organizations". En International Journal of Project Management, 23(5), p. 374-379.

SAVIOTTI, P. P. (1998). "On the dynamics of appropriability, of tacit and of codified knowledge". En Research Policy, 26(7-8), p. 843-856.

SFARD, A. (1998). "On Two Metaphors for Learning and the Dangers of Choosing Just One". En Educational Researcher, 27(2), p. 4-13.

SORENSON, O., RIVKIN, J. W., y FLEMING, L. (2006). “Complexity, networks and knowledge flow". En Research Policy, 35(7), p. 994-1017.

WENGER, E. (1998). “Communities of practice: Learning as a social system”. En Systems Thinker 9(5), p. 2-3.

6.4 Ponencias o Presentaciones en Jornadas o Congresos

COHENDET, P., CREPLET, F., y DUPOÜET, O. (2000). “Communities of practice and epistemic communities: a renewed approach of organisational learning within 
the firm". En Proceedings of the 5th Workshop on Economics with Heterogeneous Interacting Agents (WEHIA), Marseille, 15-17 de Julio de 2000.

HOCHGERNER, J. (2012): “Thoughts about Social Innovation". Powerpoint de la Ponencia presentada en la Escuela de Verano "La innovación social y sus agendas" celebrada en Donostia-San Sebastián los días 2, 3 y 4 de julio de 2012.

\section{El autor}

Alfonso Unceta es doctor en Sociología y Profesor Titular de Sociología de la UPV/EHU donde ha desarrollado la mayor parte de su trayectoria profesional. Fruto de su tarea investigadora ha participado en más de treinta proyectos de investigación y contratos externos. Es asimismo autor de más de cincuenta publicaciones (libros, libros colectivos y artículos en revistas especializadas). Sus líneas prioritarias de trabajo son Educación, Innovación Social e Industrias Culturales y Creativas.

Ha obtenido el Premio Euskoiker de Investigación y el Premio de Investigación Jesús María Leizaola ambos en el año 2004. Entre 2007 y 2013 ha sido Director del Master en Gestión de la Innovación y el Conocimiento. En la actualidad dirige el Master en Innovación Social e Industrias Culturales y Creativas de la UPV-EHU. Es también director del centro Sinnergiak Social Innovation (UPV-EHU). 